\title{
Efecto del Programa Psicoeducativo ProFamille en el grado de sobrecarga y síntomas psíquicos en familiares cuidadores de personas enfermas de esquizofrenia Effect of the ProFamille Psychoeducational Program on the degree of burden of care and psychological symptoms in family caregivers of people with schizophrenia
}

Franco Cid L. ${ }^{1}$ y Alvaro Cavieres F. ${ }^{1}$

\begin{abstract}
Most people with schizophrenia continue their treatment within their families. This can lead to a deterioration of the life quality of their caregivers, by compromising economic and social aspects, as well as their physical and mental health. Evidence shows that psychosocial interventions can alleviate this burden of care. In Chile, the Ministry of Health recommends the inclusion of psychosocial interventions in the treatment of schizophrenia patients but does not propose any specific programs. The purpose of this research is to examine the effect of the program Profamille on the severity of burden of care and psychological symptoms of caregivers of patients with schizophrenia. Subjects and Methods: Open, non-controlled clinical trial. The program was carried out in rehabilitation centers in three Chilean cities for a period of five months. Participants included 51 caregivers of people with schizophrenia with at least one year of evolution and ambulatory treatment. The program consisted of 10 biweekly, 2.5 hours long sessions led by a clinical psychologist and a psychiatric resident. Self-evaluation scales were used to assess the results. Results: The program dropout rate was 31\%. Mean scores showed significant differences in the scales of depression and anxiety, pre and post program, with a moderate effect size. There were no differences in the scale of burden of care. Discussion: The Profamille program shows ease of implementation, cost-effectiveness, and a potential favorable effect. Further examination of this program with medium and long term follow-up is suggested.
\end{abstract}

Key words: Psychosocial interventions, schizophrenia, Profamille.

Rev Chil Neuro-Psiquiat 2013; 51 (2): 102-109

\section{Introducción}

$\mathrm{E}$ avance de la terapéutica y la desinstitucionalización, han hecho que un número creciente de personas enfermas de esquizofrenia, continúen

Recibido: 27/12/2012

Aceptado: 22/05/2013

Los autores no presentan ningún tipo de conflicto de interés.

1 Departamento de Psiquiatría, Universidad de Valparaíso. su tratamiento en la comunidad, la mayoría de las veces, a cargo de sus familias ${ }^{1}$, situación que se presenta con más frecuencia en nuestra cultura ${ }^{2,3}$.

El fenómeno descrito ha llevado a reconocer oficialmente, el rol de los familiares en el cuidado 
de los pacientes ${ }^{4,5}$, y sus propias necesidades de apoyo $^{6}$. Sin embargo, diversos estudios muestran un deterioro significativo en la calidad de vida de los cuidadores, comprometiendo aspectos económicos, sociales y de salud física y mental ${ }^{7-9}$. Los principales factores que interactúan al respecto, son las características personales y distintos estresores sociales, situacionales y iatrogénicos ${ }^{10}$.

Un concepto clave para comprender lo anterior, es el de Sobrecarga del Cuidador, definido por Dillehay y Sandys ${ }^{11}$, como "un estado psicológico resultante de la combinación del trabajo físico, la presión social y emocional y las restricciones económicas derivadas del cuidado de los pacientes". En el caso de la esquizofrenia, diversos estudios, reportan altos niveles de sobrecarga, en al menos, un tercio de los cuidadores ${ }^{12-14}$, especialmente mujeres, quienes habitualmente realizan esta labor ${ }^{15,16}$. Los síntomas psicóticos ${ }^{17,18}$, y los deficitarios ${ }^{19}$, una menor compensación y nivel de funcionamiento psicosocial de los pacientes, han sido asociados con una mayor sobrecarga ${ }^{20}$, así como a mayores costos médicos, cesantía y dependencia económica ${ }^{21,22}$.

También se ha informado que alrededor de un $25 \%$ de los cuidadores de pacientes con esquizofrenia, presentan altos niveles de síntomas anímicos y $\operatorname{ansiosos}^{23-26}$. Las variables más comunes, relacionadas con la depresión fueron el género femenino, nivel socioeconómico bajo, padres separados o solteros, tamaño familiar reducido, educación deficiente, estrategias de afrontamiento insuficientes, pobre relación con el paciente, y el estigma asociado al trastorno mental ${ }^{27}$.

En Chile, dos estudios realizados en el norte del país empleando la escala de sobrecarga de Zarit, reportan niveles de sobrecarga intensa, en sobre el $90 \%$ de los cuidadores ${ }^{15,28}$. Los autores explican estos hallazgos a partir de la pobreza y falta de acceso a dispositivos de rehabilitación de los entrevistados. Significativamente, usando el mismo instrumento, en una muestra de Santiago, se informa alrededor de un $50 \%$ de cuidadores en esta condición ${ }^{29}$.

El término psicoeducación, introducido en 1980 por Anderson ${ }^{30}$, designa un tipo específico de intervención, que busca educar acerca de la enfermedad, entregar herramientas para la resolución de conflictos, entrenar habilidades para una mejor comunicación y fomentar el autoestima ${ }^{31}$. Este tipo de programas, dirigido a los familiares de las personas enfermas de esquizofrenia, ha demostrado aumentar su nivel de conocimientos acerca de la enfermedad, mejorar su calidad de vida, adecuar las actitudes y expectativas hacia el paciente ${ }^{32} \mathrm{y}$ facilitar también, la relación médico-familiares ${ }^{33}$.

El programa "ProFamille" es una intervención psicosocial con estas características. Concebido por el Prof. Hugo Cormier de la Universidad de Quebec, en $1991^{34}$, su propósito es intentar evitar que los cuidadores sufran un menoscabo en su calidad de vida y ayudar a que puedan desarrollarse como individuos, pese a su responsabilidad. Bajo esta óptica, pretende ser una herramienta, entre otras, que posibilita alcanzar un sistema de vida armónico y saludable para toda la familia. Consta de 10 bloques temáticos, que incluyen información respecto a las causas y tratamiento de la enfermedad, desarrollo de habilidades de comunicación, establecimiento de límites, autovaloración y la búsqueda y empleo de ayuda.

El programa abarca tres dimensiones: 1) Pedagógica: a través de la entrega de información acerca de la enfermedad; 2) Psicológica: en que se considera conocer el diagnostico, la liberación de la carga emocional, y el duelo debido a las pérdidas relacionadas con la enfermedad; 3) terapéutica conductual: en que se aplican estrategias para lograr cambios de algunas conductas. Las investigaciones desarrolladas hasta ahora, muestran un buen nivel de aceptación por los participantes, un mejoramiento en su nivel de conocimientos y habilidades y una reducción en sus niveles de malestar psíquico $^{35,36}$.

A pesar de sus beneficios, estudios europeos muestran que los programas psicoeducativos no son comúnmente aplicados en la práctica clínica, posiblemente debido a la falta de capacitación ${ }^{14,37,38}$. En Chile, en tanto, el Ministerio de Salud recomienda altamente, incluir intervenciones familiares en el tratamiento de toda persona con esquizofrenia y en todas las fases de la enfermedad, sin embargo, no se proponen programas específicos a implementar ${ }^{39}$. 
En nuestro país, las publicaciones respecto a la implementación de programas de psicoeducación familiar son más bien escasas, una de ellas evaluó el impacto en la sobrecarga usando la escala de Zarit y desglosándola en 3 subítems: carga, rechazo e incompetencia. Se encontró una reducción significativa de la sobrecarga, especialmente en madres y cuidadores con bajo nivel educacional ${ }^{40}$.

El propósito de esta investigación, es realizar una intervención exploratoria, para conocer el efecto del programa Profamille en el grado de sobrecarga y sintomatología depresiva y ansiosa, en una muestra de familiares cuidadores de pacientes con esquizofrenia atendidos en forma ambulatoria en el Policlínico del Hospital del Salvador de Valparaíso.

\section{Sujetos y Método}

Este es un ensayo clínico abierto, exploratorio, no controlado, de tipo pre-post. El programa Profamille se llevó a cabo, durante cinco meses, en tres centros de rehabilitación pertenecientes a la Corporación Bresky, en distintas ciudades de la quinta región; Valparaíso, Viña del Mar y Peñablanca y en el Hospital Psiquiátrico del Salvador de Valparaíso.

\section{Sujetos}

Se incluyó un total de 51 cuidadores, familiares de personas con diagnóstico de esquizofrenia, (un familiar por paciente), con al menos un año de evolución, en tratamiento ambulatorio y asistentes a uno de los cuatro centros. La muestra incluye aproximadamente, la misma cantidad de pacientes por cada centro. Los sujetos participantes fueron invitados a participar, en forma secuencial, al momento de acompañar a sus familiares a sus controles médicos habituales o actividades de rehabilitación. Todos los voluntarios entregaron su consentimiento informado por escrito. Todos los pacientes fueron diagnosticados según los criterios del DSM IV y CIE 10. Una descripción de la muestra se presenta en las Tablas 1 y 2.

\section{Intervención psicoeducativa}

El programa Profamille consta de 10 sesiones bisemanales de aproximadamente 2 horas y media
Tabla 1. Características socio demográficas de la muestra de cuidadores

\begin{tabular}{|lc|}
\hline Edad en años, media \pm DE (rango) & $56,7 \pm 11,3(25-78)$ \\
Género n (\%) & \\
Femenino & $41(88,6)$ \\
Masculino & $(11,4)$ \\
Estado civil n & $19(54,3)$ \\
Casado/a & $7(20)$ \\
Soltero/a & $6(17,1)$ \\
Viudo/a & $1(2,9)$ \\
Convive & $2(5,7)$ \\
Separado/a & \\
Nivel educacional n $(\%)$ & $5(14,3)$ \\
Básico & $20(57,1)$ \\
Medio & $10(28,6)$ \\
Superior & \\
Parentesco n $\%)$ & $29(82,9)$ \\
Padre/Madre & $4(11,4)$ \\
Hermano/a & $2(5,8)$ \\
Otro & $7(20)$ \\
Trabajo fuera de casa &
\end{tabular}

Tabla 2. Características socio demográficas de la muestra de pacientes

\begin{tabular}{|c|c|}
\hline Edad en años, media \pm DE (rango) & $33,7 \pm 11,7(15-60)$ \\
\hline $\begin{array}{c}\text { Género n (\%) } \\
\text { Femenino } \\
\text { Masculino }\end{array}$ & $\begin{array}{r}9(25,71) \\
26(74,29)\end{array}$ \\
\hline $\begin{array}{l}\text { Nivel educacional n (\%) } \\
\text { Básico } \\
\text { Medio } \\
\text { Superior }\end{array}$ & $\begin{aligned} & (20) \\
23 & (65,71) \\
5 & (14,29)\end{aligned}$ \\
\hline $\begin{array}{l}\text { Edad en años al diagnóstico, } \\
\text { media } \pm \text { DE (rango) }\end{array}$ & $20,9 \pm 4,1(13-30)$ \\
\hline $\begin{array}{l}\text { Hospitalización últimos dos años } \\
\text { n (\%) }\end{array}$ & $4(11,43)$ \\
\hline $\begin{array}{l}\text { Asistencia a dispositivo de } \\
\text { rehabilitación } \mathrm{n}(\%)\end{array}$ & $21(60)$ \\
\hline $\begin{array}{l}\text { Antipsicótico n (\%) } \\
\text { Risperidona } \\
\text { Clozapina } \\
\text { Otros atípicos } \\
1^{\circ} \text { Generación }\end{array}$ & $\begin{array}{c}19(54,2) \\
9(25,71) \\
3(8,57) \\
4(11,43)\end{array}$ \\
\hline $\begin{array}{l}\text { Uso de NL depot n (\%) } \\
\text { Otro }\end{array}$ & $\begin{array}{l}6(17,14) \\
2(5,8)\end{array}$ \\
\hline Trabajo fuera de casa & $7(20)$ \\
\hline
\end{tabular}


de duración. Los contenidos expuestos son: 1) Recepción y Bienvenida; 2) Esquizofrenia: causas, efectos y pronóstico; 3) Tratamiento de la esquizofrenia; 4) Habilidades de comunicación; 5) Habilidades para poner límites; 6) Habilidades para desarrollar autovaloración y autoconfianza; 7) Manejo de sentimientos de culpa; 8) Habilidades para desarrollar expectativas realistas; 9) Habilidades para utilizar la ayuda necesaria y 10) Habilidades para construir y cuidar una red social.

Un manual describe en detalle el desarrollo de cada sesión, además propone diversos textos para presentar y discutir las temáticas, incorporando la modalidad de "role playing" y dinámicas de grupo. Las sesiones fueron conducidas por dos profesionales de salud mental, psiquiatras, residentes de psiquiatría y/o psicólogos entrenados en el programa. Cada grupo fue integrado por entre $10 \mathrm{a}$ 12 personas.

\section{Mediciones}

Todos los sujetos participantes completaron las siguientes encuestas autoaplicadas, en modalidad lápiz y papel, al inicio y al finalizar el programa:

Escala de Sobrecarga del cuidador de Zarit ${ }^{41}$ : Consta de 22 preguntas que evalúan las repercusiones de la prestación de cuidados en cuatro ámbitos de la vida del cuidador; salud física, salud psíquica, actividades sociales y recursos económicos. Los valores de las respuestas oscilan entre 1 y 5 según una escala de Likert y los resultados se suman en un puntaje total (22-110 puntos). El puntaje de corte propuesto es 45 . Dando 3 categorías: sin sobrecarga (22 a 46), sobrecarga moderada (47 a 55) y sobrecarga intensa (56 a 110). Este instrumento ha sido validado en nuestro país ${ }^{29}$.

Escala para la Depresión de Zung y Conde ${ }^{42}$ : Consta de 20 preguntas, formuladas la mitad, en términos positivos y el resto en términos negativos. Se agrupan en 4 factores; depresivo, biológico, psicológico y psicosocial. Cada una, se responde con un puntaje de uno a cuatro, utilizando una escala Likert, según la frecuencia de presentación del síntoma. Proporciona una puntuación total de gravedad, con un puntaje de corte de 35 y 3 categorías: leve (36-51), moderada (52-67) y grave (mayor a 68). El marco de referencia temporal es el momento actual. Validada en Chile por Conde et al en $1970^{43}$.

Escala para la Ansiedad Autoaplicada de Zung ${ }^{44}$. La escala mide el nivel de ansiedad en personas que experimentan síntomas de angustia. Consta de 20 preguntas, cada una se responde con un puntaje de uno a cuatro, según la frecuencia de presentación de la sintomatología. Proporciona una puntuación total de gravedad, con un puntaje de corte de 44, y tres categorías: leve a moderado (45-79), severo (60-74) y extrema (75-80). El marco de referencia temporal es el momento actual. Esta escala se encuentra validada en su versión en castellano ${ }^{45}$.

Adicionalmente se completó una encuesta con datos clínicos y demográficos tanto del paciente como de su cuidador.

\section{Análisis estadístico}

Se realiza un análisis descriptivo con los datos generales de cada paciente y de su cuidador.

Se aplica la prueba normalidad de Kolmogorov Smirnov a los puntajes totales sobre ansiedad, depresión y sobrecarga obtenidos pre y post aplicación del programa Profamille. La comparación de los puntajes totales, grupal, pre y post aplicación del programa Profamille se realizó con una prueba de hipótesis para diferencia de medias para muestras dependientes, que proporciona un estadístico $\mathrm{t}$ de Student y un valor p, considerando un $95 \%$ de confianza, error de alpha de 0,05. El software estadístico para análisis empleado es MINITAB.

\section{Resultados}

La muestra inicial fue de 51 cuidadores, siendo el porcentaje de abandono del programa de un $31 \%$ (16 sujetos), por lo que la muestra final incluyó un total de 35 cuidadores.

En cuanto a los resultados en las diferencias de medias pre y post programa, se observan diferencias significativas en las escalas de depresión y ansiedad, no así en la escala de sobrecarga (Tabla 3). El tamaño del efecto, según la d Cohen, en ambos casos fue de 0,4, es decir, moderado. En el caso de 
Tabla 3. Mediciones pre y post intervención

\begin{tabular}{|lcccc|}
\hline Escala & $\begin{array}{c}\text { Pre-taller } \\
\text { Media } \pm \text { DE }\end{array}$ & $\begin{array}{c}\text { Post-taller } \\
\text { Media } \pm \text { DE }\end{array}$ & Valor $\mathbf{p}$ & Tamaño del efecto \\
Depresión (Zung y Conde) & $42,5 \pm 9,9$ & $38,8 \pm 8,7$ & 0,005 & 0,4 \\
Ansiedad (Zung) & $39,9 \pm 12,4$ & $35,5 \pm 10,9$ & 0,008 & 0,4 \\
Sobrecarga (Zarit) & $58,4 \pm 16,2$ & $54,2 \pm 16,2$ & 0,101 & 0,25 \\
\hline
\end{tabular}

la sobrecarga, el tamaño del efecto fue de 0,25 , lo que equivale a un efecto pequeño.

El análisis por categorías, muestra que un 16 $(45,7 \%)$ y $8(22,9 \%)$ de los participantes presentaron depresividad leve y moderada antes del taller, y $15(42,9 \%)$ y $3(8,6)$ después de este. En el caso de la ansiedad, la distribución pre taller fue de 4 $(11,4 \%)$ para síntomas leves a moderados y de 4 $(11,4 \%)$ para los severos, post taller, los resultados fueron de $3(8,6 \%)$ y $3(8,6 \%)$. Respecto a la medición de sobrecarga pre taller, 7 (20\%) presentaron un nivel leve, y 19 (54,3\%) intenso, posterior al taller, $3(8,6 \%)$ presentó sobrecarga leve y 17 (48,6\%) continuaba presentando sobrecarga intensa.

\section{Discusión}

Si bien el propósito fundamental de esta investigación es exploratorio, sus resultados muestran un efecto favorable del programa Profamille, en los síntomas depresivos, pero no así en la sobrecarga. Esto último no sorprende, dado que revisiones recientes, no encuentran un efecto positivo concluyente de la psicoeducación en este ámbito ${ }^{46}$. No obstante, otros ensayos si lo reportan ${ }^{15,40,47}$.

El efecto sobre la sintomatología depresiva y ansiosa se podría explicar a partir del componente psicoterapéutico de este programa, dirigido a la superación de las dificultades y problemas personales asociados al rol de cuidador, por el contrario, la sobrecarga pudiera estar más condicionada, aparte del cuidado del paciente en sí mismo, por factores desfavorables de índole socio-económicos.

Aunque este estudio, no lo evaluó dirigidamente, se puede inferir que la muestra, usuarios del sistema público de salud, presenta un nivel socio-económico medio a medio bajo, agravando la sobrecarga con la carencia de recursos y redes de apoyo. Esto concuerda con otro estudio realizado en nuestro país, al comprobar que la sobrecarga se presentaría en alto grado, sobre todo en cuidadoras madres, de bajo nivel educacional, desempleadas, $y$ a cargo de pacientes jóvenes ${ }^{15}$.

No se observan diferencias clínicas ni demográficas entre quienes completaron el programa y quienes desertaron, como tampoco entre quienes obtuvieron beneficios y los que no lo hicieron. La alta tasa de abandono observado (31\%), principalmente por motivos económicos, es una limitante significativa para programas de este tipo, por lo mismo, sería importante evaluar versiones breves $\mathrm{o}$ intensivas.

Existen varias limitaciones en este estudio. La muestra, si bien incluyó a 3 comunas de la región, puede no ser representativa de la población general. El diseño no incluyó un grupo control, por la falta de disponibilidad de un mayor número de participantes, fundamentalmente por motivos económicos. Por otra parte, la evaluación post taller, se realizó inmediatamente al término del mismo, habiendo sido más apropiada una reevaluación al mediano plazo. Finalmente, las conclusiones deben ser tomadas con cautela, ya que el tamaño de la muestra limita la obtención de conclusiones más contundentes.

Se requieren más ensayos con este Programa con seguimientos de mediano y largo plazo, ya que resulta conveniente por su costo-efectividad, facilidad de implementación y con un potencial efecto favorable, no sólo en la familia, sino también en los pacientes y sistema público de salud al aportar con nuevas prestaciones que ayudan a disminuir las rehospitalizaciones y enriquecen el quehacer profesional en el ámbito de la salud mental. 


\section{Resumen}

La mayoría de las personas enfermas de esquizofrenia, continúan su tratamiento a cargo de sus familias. Esto lleva al deterioro en la calidad de vida de sus cuidadores, comprometiendo aspectos económicos, sociales y de salud física y mental. La evidencia muestra que las intervenciones psicosociales, pueden aliviar esta sobrecarga. En Chile, el Ministerio de Salud, recomienda incluirlas en el tratamiento de la esquizofrenia, sin proponer programas específicos. El propósito de esta investigación, es conocer el efecto del programa Profamille, en el grado de sobrecarga y sintomatología depresiva y ansiosa de familiares de personas con esquizofrenia. Sujetos y Método: Ensayo clínico abierto, no controlado, de tipo pre-post. El Programa consistió en 10 sesiones bisemanales, de 2 horas y media de duración, dirigidas por un psicólogo clínico y un residente de psiquiatría, en centros de rehabilitación de la quinta región. Participaron 51 cuidadores de personas con diagnóstico de esquizofrenia, con al menos, un año de evolución y en tratamiento ambulatorio. El efecto del Programa fue medido con las Escalas de Sobrecarga de Zarit, Depresión de Zung y Conde y Ansiedad de Zung. Resultados: El porcentaje de abandono del programa fue un $31 \%$. Las medias de los puntajes pre y post programa, muestran diferencias significativas en las escalas de depresión y ansiedad, con un tamaño de efecto moderado, no así en la escala de sobrecarga. Discusión: Por su costo-efectividad, facilidad de implementación y potencial efecto favorable, se recomiendan más ensayos con este programa con seguimientos de mediano y largo plazo.

Palabras clave: Intervenciones Psicosociales, Esquizofrenia, Profamille.

\section{Referencias bibliográficas}

1. Rossler W, Salize H, Cucchiaro G, Reinhard I, Kernig C. Does the place of treatment influence the quality of life of schizophrenics? Acta Psychiatr Scand 1999; 100: 142-8.

2. Milstein G, Midlarsky E. Ethnic differences in the interpretation of mental illness: perspectives of caregivers. Research in Community and Mental Health 1995; 8: 155-78.

3. Guarnaccia G. Ethnicity, social status, and families' experiences of caring for a mentally ill family member. Community Mental Health Journal 1996; 32: 243-60.

4. Lehman A, Dixon L. Practice guideline for the treatment of patients with schizophrenia. Am J Psychiatry 2004; 161: 1-56.

5. Lehman A, Kreyenbuhl J, Buchanan R, et al. The Schizophrenia Patient Outcomes Research Team (PORT): updated treatment recommendations 2003. Schizophr Bull 2004; 30: 193-217.
6. Magliano L, Marasco C, Fiorillo A, Malangone C, Guarneri M, Maj M. The impact of professional and social network support on the burden of families of patients with schizophrenia in Italy. Acta Psychiatr Scand 2002;106: 291-8.

7. Magaña S, Hernández M, Cortez R. Psychological Distress Among Latino Family Caregivers of Adults With Schizophrenia: The Roles of Burden and Stigma Psychiatr Serv 2007; 58: 378-84.

8. Martens L, Addington J. The psychological wellbeing of family members of individuals with schizophrenia. Soc Psychiatry Psychiatr Epidemiol 2001; 36: 128-33.

9. Ivarsson A, Sidenvall B, Carlsson M. The factor structure of the Burden Assessment Scale and the perceived burden of caregivers for individuals with severe mental disorders. Scand J Caring Sci. 2004; 18: 396-401.

10. Simon M. Calidad de vida de los familiares de los enfermos mentales. En Calidad de vida en los trastornos mentales, Katsching H,Freeman H, Sar- 
torius N eds. Barcelona: Masson; 2000. p. 243-50.

11. Dillehay R, Sandys M. Caregivers for Alzheimer's patients: what we are learning from research. Int J Aging Hum Dev 1990; 30: 263-85.

12. Maurin J, Boyd C. Burden of mental illness on the family: a critical review. Arch Psychiatr Nurs 1990; 4: 99-107.

13. Awad A, Voruganti L. The burden of schizophrenia on caregivers: a review. Pharmacoeconomics. 2008; 26: 149-62.

14. Magliano L, Fadden G, Madianos M, et al. Burden on the families of patients with schizophrenia: results of the BIOMED I study. Soc Psychiatry Psychiatr Epidemiol 1998; 33: 405-12.

15. Caqueo A, Gutiérrez J, Kavanagh D. Burden of care in families of patients with schizophrenia. Quality of Life Research 2006; 15: 719-24.

16. Schmid R, Spiessl H, Cording C. Between responsibility and delimitation: emotional distress of caregivers. Psychiatr Prax 2005; 32: 272-80.

17. Woltraus J, Dingemans P. A Caregiver burden in recent onset schizophrenia and spectrum disorders. The influence of symptoms and personality traits J Nerv Ment Dis 2002; 190: 241-7.

18. Magliano L, Fiorillo A, Malangone C, et al. Family burden in schizophrenia: effects of socioenvironmental and clinical variables and family intervention. Epidemiol Psichiatr Soc 1998; 7: 178-87.

19. Dyck D, Short R, Vitaliano PP. Predictors of burden and infectious illness in schizophrenia caregivers. Psychosom Med 1999; 61: 411-9.

20. Parabiaghi A, Lasalvia A, Bonetto C, et al. Predictors of changes in caregiving burden in people with schizophrenia: a 3-year follow-up study in a community mental health service. Acta Psychiatr Scand Suppl 2007; 66-76.

21. Fadden G, Kuipers L. The burden of care: The impact of functional psychiatric illness on the patient's family. Br J Psychiatry 1987; 150: 285-92.

22. Hou S, Ke C, Su Y, Lung FW, Huang C. Exploring the burden of the primary family caregivers of schizophrenia patients in Taiwan. Psychiatry Clin Neurosci 2008; 62: 508-14.
23. Ashraf M, Yasser M. Depressive Disorders Among Caregivers of Schizophrenic Patients in Relation To Burden of Care and Perceived Stigma. Current Psychiatry 2010; 17: 15-25.

24. Saunders J. Families living with severe mental illness: a literature review. Issues Ment Health Nurs 2003; 24: 175-98.

25. Caqueo-Urízar A, Gutiérrez-Maldonado J, FerrerGarcía M, Peñaloza-Salazar C, Richards-Araya D, Cuadra-Peralta A. Attitudes and burden in relatives of patients with schizophrenia in a middle income country. BMC Family Practice 2011; 12: 101-13.

26. Heru A, Ryan C. Depressive symptoms and family functioning in the caregivers of recently hospitalized patients with chronic/recurrent mood disorders. Int J Psychosoc Rehabil 2002; 7: 53-60.

27. Osman C, Alipah B, Tutiiryani MD, Ainsah O. Depressive disorders and family functioning among the caregivers of patients with schizophrenia. East Asian Arch Psychiatry 2010; 20: 101-8.

28. Gutiérrez-Maldonado J, Caqueo A, Kavanagh D. Burden of care and general health in families of patients with schizophrenia. Soc Psychiatry Psychiatr Epidemiol 2005; 40: 899-904.

29. Breinbauer H, Mayanz S, Guerra C. Validación en Chile de la Escala de Sobrecarga del Cuidador de Zarit en sus versiones original y abreviada. Rev Med Chile 2009; 137: 657-65.

30. Anderson C, Hogarty G, Reiss D. Family treatment of adult schizophrenic patients: a psycho-educational approach. Schizophr Bull 1980; 6: 490-505.

31. Bauml J, Frobose T, Kraemer S, Rentrop M, Pitschel-Walz G. Psychoeducation: a basic psychotherapeutic intervention for patients with schizophrenia and their families. Schizophr Bull 2006; 32 Suppl 1: S1-9.

32. Magliano L, Fiorillo A, Malangone C, De Rosa C, Maj M. Patient functioning and family burden in a controlled, real-world trial of family psychoeducation for schizophrenia. Psychiatr Serv 2006; 57: 1784-91.

33. Belloso J GB, de Prado E. Intervención psicoeducativa en un centro de rehabilitación psicosocial. 
Rev Asoc Esp Neuropsiq 2000; 73: 23-40.

34. Cormier H Gulmond G, et al. Profamille: Programme d'Intervention de groupe auprès des families de personnes atteintes de schizophrénie. Merchlenne-au-Pont, Belgique: Socrate réhabilitation; 1993.

35. Hodé Y Krychowski R, Beck N, et al. Effet d'un programme psychoéducatif sur l'humeur des familles des malades souffrant de schizophrénie. Journal de thérapie comportementale et cognitive. 2008; 10: 1016-20.

36. Hodé Y. Prise en charge des familles de patients schizophrènes, Family intervention for schizophrenia. Annales Médico-Psychologiques 2011; 169: 196-9.

37. McFarlane W, Lukens E, Link B, et al. Multiple-family groups and psychoeducation in the treatment of schizophrenia. Arch Gen Psychiatry 1995; 52: 679-87.

38. Fadden G. Implementation of family interventions in routine clinical practice following staff training programs: a major cause for concern. J Ment Health 1997; 6: 599-612.

39. Minsal. Ministerio de Salud. Guía Clínica para el tratamiento de personas desde primer episodio de esquizofrenia. Santiago 2009.
40. Gutiérrez J, Caqueo A. Effectiveness of a psychoeducational intervention for reducing burden in latin american families of patients with schizophrenia. Qual Life Res 2007; 16: 739-47.

41. Zarit S, Reever K, Bach-Peterson J. Relatives of the impaired elderly: correlates of feelings of burden. Gerontologist 1980; 20: 649-55.

42. Zung W. A self-rating depression scale. Arch Gen Psychiatry 1965; 12: 63-70.

43. Conde V, Escriba J, Izquierdo J. Statistic evaluation and Spanish adaptation of Zung's self-applied scale for depression. Arch Neurobiol 1970; 33: 281-302.

44. Zung W. A rating instrument for anxiety disorders. Psychosomatics 1971; 12: 371-9.

45. Lusilla M, Sanz C, López J. Validación estructural de la escala heteroevaluada de ansiedad de Zung. Anales de Psiquiatría 1990; 6: 39.

46. Pharoah F, Mari J, Rathbone J, Wong W. Family intervention for schizophrenia. Cochrane Database Syst Rev 2006 (4): CD000088.

47. Sharif F, Shaygan M, Mani A. Effect of a psychoeducational intervention for family members on caregiver burdens and psychiatric symptoms in patients with schizophrenia in Shiraz, Iran. BMC Psychiatry 2012; 12: 48.
Correspondencia:

Alvaro Cavieres Fernández

9 Norte 915 Viña del Mar F: 2884850

E-mail: cavieres.alvaro@gmail.com 